Nervenarzt 2008

DOI 10.1007/s00115-008-2473-7

Online publiziert: 25 . April 2008

(c) Springer Medizin Verlag 2008

Z. Zala $\cdot$ R. Kuckelsberg ${ }^{1} \cdot$ U. Busch-Wübbena ${ }^{1} \cdot$ C. Kick ${ }^{1} \cdot$ J. Hinrichs ${ }^{1} \cdot$ A. Heinz ${ }^{2}$.

A. Ströhle ${ }^{2}$

${ }^{1}$ Max-Planck-Institut für Psychiatrie, München

2 Klinik für Psychiatrie und Psychotherapie, Charité -

Universitätsmedizin Berlin, Campus Mitte, Berlin

\title{
Patientengerechte Aggressionsbewältigung in der Psychiatrie
}

\section{Beschreibung eines Seminars und erste Ergebnisse}

Aggression und Gewalt in der Psychiatrie werden insbesondere unter dem Aspekt von Behandlungen gegen den Willen des Patienten wahrgenommen. Weniger Beachtung findet die Tatsache, dass Mitarbeiter psychiatrischer Einrichtungen möglicher Aggression und Gewalt, meist im Zusammenhang mit so genannten Erregungszuständen der Patienten, ausgesetzt sein können [2, 4, 10, 15, 16, 19]. Diese Gewalt führt zu einer beträchtlichen Anzahl von Arbeitsunfällen sowie Angst und Unsicherheit im Team. Patientenübergriffe sind nach einer Auswertung des Gemeindeunfallversicherungsverbands Westfalen-Lippe mit etwa 40\% die häufigsten Arbeitsunfälle [14].

Während verbale Deeskalationsstrategien in vielen Fort- und Weiterbildungen in der Psychiatrie vermittelt werden, wird der praktische Umgang mit tätlichen Angriffen bislang kaum gelehrt. Inzwischen wurden jedoch erste Trainingsprogramme zum praktischen Umgang mit aggressiven Patienten entwickelt [3]. Bei Pflegeschülern führte ein 4-tägiges Training zum Umgang mit Aggression und Gewalt zu einem Anstieg der Selbstwirksamkeit [17]. Während aggressives und gewalttätiges Verhalten durch eine systematische Risikoanalyse und einen Trainingskurs nicht beeinflusst wurde, fand sich eine Reduk- tion von Zwangsmaßnahmen [8]. In einer systematischen Übersicht kommen Richter und Needham zu dem Schluss, dass es bisher noch keine klare Evidenz für eine Reduktion gewalttätiger Übergriffe gibt. Eindeutig belegt seien jedoch positive Effekte auf das Wissen über Gewalt und das Vertrauen, mit diesen schwierigen Situationen umgehen zu können [14].

Auf der Basis eines handlungstheoretischen Partialmodells konnten Kompetenz- und Kontrollüberzeugungen herausgearbeitet werden, die zur Beschreibung, Rekonstruktion und Vorhersage von Handeln geeignet sind. Ausgehend von Arbeiten mit dem in den 196oiger Jahren vorgelegten IPC-Fragebogen $[8,9]$ wurde im deutschen Sprachraum von Krampen [7] der Fragebogen zu Kompetenz- und Kontrollüberzeugung (FKK) entwickelt und validiert. Studien nach Einführungskursen zu autogenem Training oder nach Therapie depressiver Störungen belegen die Veränderungssensitivität des FKK [7]. Nach einem Seminar, das einen aktiv autonomen, von Selbstsicherheit und sicherer Handlungsplanung und -durchführung geprägten Umgang mit Aggression und Gewalt anstrebt, ist ein Anstieg von internaler Kontroll- und Kompetenzüberzeugung zu erwarten.
Nachfolgend wird das Programm „Patientengerechte Aggressionsbewältigung in der Psychiatrie" beschrieben, die Beurteilung durch die Teilnehmer dargestellt und in einer Teilstichprobe untersucht, inwiefern das Trainingsseminar zu (überdauernden) Veränderungen von Kompetenz- und Kontrollüberzeugungen bei den Teilnehmern führt.

\section{Methodik}

\section{Beschreibung des Seminars}

Das Seminar „Patientengerechte Aggressionsbewältigung in der Psychiatrie“ (PAP) wurde für Mitarbeiter der verschiedenen Berufsgruppen psychiatrischer oder sozialer Einrichtungen entwickelt. Es setzt keine Vorkenntnisse in Selbstverteidigung oder Fitness voraus. Folgende Inhalte werden thematisiert:

- Formen von Aggression: Selbst-, Fremd-, verbale-, passive Aggression,

- Entstehung von Aggression und Angst; Angst im Berufsalltag,

- Verhaltensweisen in aggressionsbelasteten Situationen,

- Eskalationsspirale und (verbale) Deeskalation,

- Umgang mit Gewalt, 
- ethische Grundlagen des Fremd- und Selbstschutzes,

- rechtliche Aspekte,

- sichere und schnell erlernbare Selbstverteidigungstechniken.

Zentral ist jedoch die Vermittlung und Einübung einfacher Selbstverteidigungstechniken mit Elementen, die aus fernöstlichen Kampfkunstarten (Jiu-Jitsu, Aikido, Kung-Fu) ausgesucht und zusammengestellt wurden und die den höchstmöglichen Wirkungsgrad im Hinblick auf die Abwehr körperlicher Aggression und Gewalt ermöglichen. Neben der einfachen Vermittelbarkeit erfolgte die Auswahl der Techniken mit dem Ziel, die Verletzungsgefahr für beide Parteien (Klinikpersonal und aggressive Patienten) soweit wie möglich zu minimieren.

Die praktischen Übungen haben folgende Inhalte:

- eine kurze, aber gründliche theoretische Aufklärung über Grundregeln und Grundkenntnisse der Selbstverteidigung,

- Blocktechniken, die die lebenswichtigen Körperregionen (Kopf und Rumpf) bei einem Angriff schützen,

- Abwehrtechniken, die helfen, den Angriff effektiv abzuwehren und den Angreifer unter Kontrolle zu bringen,

- Festhaltetechniken, die nach erfolgreicher Abwehr angewendet werden, um den Angreifer fixieren zu können,

- Transporttechniken, mit deren Hilfe man den Angreifer ohne großen Kraftaufwand abführen kann.

Didaktische Vorgehensweise:

- Das Seminar besteht aus 5 aufeinander aufbauenden Lehreinheiten, die jeweils $1,5 \mathrm{~h}$ dauern (entspricht insgesamt etwa einem Arbeitstag).

- Die Techniken werden besprochen und die einzelnen Schritte erläutert, demonstriert und anschließend unter Anleitung geübt.

- Für die Wiederholung einzelner Techniken, die etwas komplizierter sind, sowie für Fragen und Antworten sind jeweils 15 min pro Lehreinheit einkalkuliert.

- Das Seminarprogramm ist detailliert in einem Manual zusammengefasst.

\section{Kursteilnehmer}

Insgesamt 339 Teilnehmer nahmen zwischen 2001 und 2005 an 31 Seminaren teil. 19 Seminare fanden an 2 Tagen und 12 an 5 Tagen statt. An folgenden Einrichtungen wurde mindestens einmalig ein Seminar durchgeführt: Max-Planck-Institut für Psychiatrie, München; Klinik für Psychiatrie und Psychotherapie, Campus Charité Mitte, Berlin sowie Ludwig-Maximilians-Universität, München und Katholischer Männerfürsorgeverein, München; Krisendienst Ost, München; Tandem BQ GmbH-Ambulante Dienste, Berlin. In München werden seit Ende 2003 regelmäßig Seminare für Mitarbeiter aus sozialen Einrichtungen angeboten.

\section{Teilnehmerzufriedenheit}

Die Zufriedenheit der Teilnehmer wurde am Ende des Seminars für die Vermittlung theoretischen bzw. praktischen Wissens, die praktische Anwendbarkeit, den Aufbau und eine Gesamteinschätzung mit Hilfe von "Schulnoten“ (1 sehr gut, 6 unbefriedigend) bewertet. 335 Teilnehmer füllten diese Fragebögen aus.

\section{Kompetenz- und Kontrollüberzeugung}

Der von Krampen [5, 6] entwickelte „Fragebogen zu Kompetenz- und Kontrollüberzeugungen“ (FKK) wurde als etabliertes und veränderungssensitives Verfahren zur standardisierten Untersuchung und Charakterisierung von Kontrollüberzeugungen angewendet. Konzeptuell und auswertungstechnisch werden im FKK 3 Aspekte generalisierter Kontrollüberzeugungen unterschieden und als Primärskalen abgebildet:

- Internalität (I) als die subjektiv bei der eigenen Person wahrgenommene Kontrolle über das eigene Leben und über Ereignisse in der personenspezifischen Umwelt,

- sozial bedingte Externalität (P powerful others control orientation) als generalisierte Erwartung, dass wichtige Ereignisse im Leben vom Einfluss anderer („mächtiger“) Personen abhängen.
- fatalistische Externalität (C chance control orientation) als generalisierte Erwartung, dass das Leben und Ereignisse vom Schicksal, Glück, Pech und dem Zufall abhängen

Als 4. Primärskala wird im FKK das

- Selbstkonzept eigener Fähigkeiten (SK) als generalisierte Erwartung darüber, dass in Handlungs- oder Lebenssituationen Handlungsmöglichkeiten zumindest eine - zur Verfügung stehen.

Neben diesen Primärskalen bietet der FKK auch aus diesen Skalen abgeleitete, auswertungstechnisch somit von den Primärskalen direkt abhängige Sekundär- und Tertiärskalen. In den beiden Sekundärskalen werden die Items der Primärskalen zu konzeptuell globaleren Indikatoren selbstbezogener Kognitionen aggregiert; die Tertiärskala resultiert aus dem Differenzwert dieser Sekundärskalen und es resultiert damit - noch globaler und konzeptuell unscharf - eine unidimensional, bipolare Persönlichkeitsdimension.

Die 1. Sekundärskala fasst die Items der Primärskalen zum Selbstkonzept eigener Fähigkeiten und zur Internalität und wird nach Bandura [1] als Dimension der generalisierten Selbstwirksamkeitsüberzeugungen (SKI) bezeichnet. Parallel dazu wird die 2. Sekundärskala durch die Aggregation der Primärskalen zur sozialen Externalität und zur fatalistischen Externalität gebildet und wird als Dimension der generalisierten Externalität in Kontrollüberzeugungen (PC) bezeichnet. Konzeptuell unschärfer ist die Tertiärskala des FKK angelegt; sie wird durch den einfachen Differenzwert von SKI-PC gebildet, umfasst somit alle 32 Items des Fragebogens und wird als globale, bipolare Dimension der generalisierten Internalität vs. Externalität in Kontrollüberzeugungen (SKI-PC) bezeichnet. Da sämtliche theoretischen und konzeptionellen Differenzierungen, die hinter der Konstruktion des FKK stehen, verwischt oder negiert werden, wird empfohlen, diese nicht im Vordergrund des Auswertung stehen soll.

Von 31 Seminarteilnehmern und 11 Kontrollprobanden wurde der Fragebo- 
gen zu Kompetenz- und Kontrollüberzeugungen vor, am Ende und 3 Monate nach dem Seminar ausgefüllt. Dargestellt werden die Mittelwerte und die Standardabweichung der Primär-, Sekundär- und Tertiärskalen des FKK.

\section{Statistische Analyse:}

Die statistische Analyse des FKK erfolgte mit einer multivariaten Varianzanalyse mit „Seminarteilnahme“ als „Zwischen-“ und „Zeit“ als „Innerhalb der Personen-“ Faktor. Bei signifikanten Hauptoder Interaktionseffekten $(\mathrm{p}<0,05)$ wurden Kontrasttests berechnet. Soziodemographische Variablen wurden mittels univariater Varianzanalyse bzw. $\chi^{2}$-Test verglichen.

\section{Ergebnisse}

\section{Teilnehmerzufriedenheit}

Das Alter der 335 Teilnehmer des Seminars die eine subjektive Einschätzung des Programms abgaben lag zwischen 19 und 62 Jahre mit einem Durchschnittsalter von 32,7 Jahren; 64\% der Teilnehmer waren Frauen, 36\% Männer. 67\% der Teilnehmer waren in der Krankenpflege tätig, 11\% waren Ärzte und 22\% anderen Berufsgruppen zugehörig (Psychologen, Lehrer, Sozialarbeiter, Ergotherapeuten, Heilpädagogen); die Rücklaufquote betrug 98,8\%

Die Vermittlung theoretischen Wissens wurde im Mittel benotet mit der Schulnote 1,44 , die Vermittlung praktischen Wissens mit 1,17, der Aufbau des Seminars mit 1,20, die Anwendbarkeit bei der Arbeit mit 1,74. Insgesamt wurde das Seminar mit 1,31 bewertet. $74 \%$ der Teilnehmer wünschten sich einen Wiederholungsbzw. einen Aufbaukurs.

\section{Kompetenz- und \\ Kontrollüberzeugung}

Das mittlere Alter der 31 mit dem FKK untersuchten Teilnehmer des Seminars betrug 34,o (SEM=1,7) Jahre. Unter den 19 Frauen und 12 Männer waren 18 Krankenpflegemitarbeiter, 3 Ärzte sowie 10 aus anderen Berufsgruppen (5 Psychologen, 3 Einzelfallhelfer und 2 medizinisch technische Assistenten). Das mittlere Al-

Nervenarzt 2008 DOI 10.1007/s00115-008-2473-7

(c) Springer Medizin Verlag 2008

\section{Z. Zala · R. Kuckelsberg · U. Busch-Wübbena · C. Kick · J. Hinrichs · A. Heinz · A. Ströhle Patientengerechte Aggressionsbewältigung in der Psychiatrie. Beschreibung eines Seminars und erste Ergebnisse}

\section{Zusammenfassung}

Gewalt und Aggression können insbesondere im Umgang mit psychiatrisch erkrankten Patienten zu Verletzungen bei Mitarbeitern führen. Mit der patientengerechten Aggressionsbewältigung wurde ein praxisorientiertes Seminar entwickelt, das den ethischen Aspekten im Umgang mit Patienten Rechnung trägt. Nach einer Beschreibung des Programms werden erste Ergebnisse einer Beurteilung durch die Teilnehmer dargestellt. Über das Seminar hinausgehende Effekte wurden mit dem Fragebogen zu Kompetenzund Kontrollüberzeugungen (FKK) untersucht. Die Teilnehmer zeigten eine sehr hohe Zufriedenheit mit dem Kurs und der Anwendbarkeit in der klinischen Praxis. Darüber hinaus fand sich bei den 31 mit dem FKK untersuchten Probanden direkt nach dem Se- minar, aber auch noch 3 Monate nach dessen Ende ein Anstieg der die aktiven und internalen Kontrollüberzeugungen anzeigenden FKK-Tertiärskala. Bei der nicht am Seminar teilnehmenden Kontrollgruppe gab es keine signifikanten Veränderungen der FKK-Skalenwerte. Ein Seminar zur patientengerechten Aggressionsbewältigung in der Psychiatrie kann zur Zufriedenheit der Teilnehmer durchgeführt werden und hat überdauernde Effekte auf Kompetenz- und Kontrollerleben.

\section{Schlüsselwörter}

Gewalt · Aggression · Psychiatrie - Seminar . Selbstschutz · Kontrollüberzeugung $\cdot$ Fragebogen zu Kompetenz- und Kontrollüberzeugungen

\section{Patient-oriented management of aggression and violence in psychiatry. Description of a training program and first results}

\section{Summary}

Violence, aggression, and patient assaults result in a considerable number of jobrelated accidents in psychiatry. We describe a patient-oriented training program for managing aggression and violence in psychiatry. Ratings by 335 training participants indicate high to very high acceptance. In addition, ratings in the questionnaire for the study subjects' beliefs about competence and control suggest a statistically significant increase in active behavior and internal locus of control in 31 subjects. No significant changes were observed in the waiting-list control group. Our results suggest that a patient-oriented training program for the management of aggression and violence in psychiatry can have high acceptance and lead to lasting changes in beliefs about competence and control, resulting in an increased internal locus of control.

\section{Keywords} Aggression - Violence - Training program . Self-defense $\cdot$ Locus of control 
Tab. 1 Mittelwerte der Primär-, Sekundär- und Tertiärskalen des Fragebogens zu Kompetenz- und Kontrollüberzeugungen bei den Seminarteilnehmern und der Kontrollgruppe

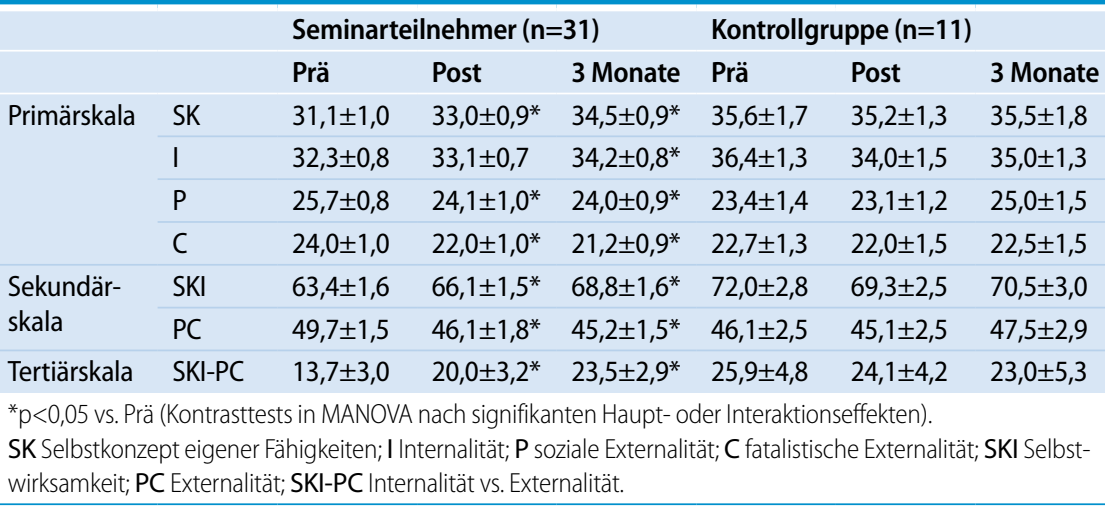

ter der Kontrollgruppe (3 Frauen, 8 Männer) betrug 35,4 \pm 3 ,o Jahre. Die Kontrollgruppe setzt sich aus 5 Krankenpflegemitarbeitern, einem Arzt sowie 4 Personen aus anderen Berufsgruppen (2 Psychologen, ein Medizinstudent und ein Sozialarbeiter) zusammen. Die Gruppen unterschieden sich nicht im Hinblick auf soziodemographische Variablen $(\mathrm{p}>0,1)$.

Die deskriptiven Statistiken der verschiedenen Skalen des FKK sind in - Tab. 1 dargestellt. Die MANOVA ergab signifikante Haupt- und Interaktionseffekte für die Primärskalen Selbstkonzept eigener Fähigkeit (SK) (Zeit: $\mathrm{F}=3,8 \mathrm{o}, \mathrm{df}=2,39$, $\mathrm{p}=0,031$; Zeit $\times$ Training: $\mathrm{F}=4,14, \mathrm{df}=2,39$, $\mathrm{p}=0,023)$, Internalität $(\mathrm{I})$ (Zeit $\times$ Training: $\mathrm{F}=4,38, \mathrm{df}=2,39, \mathrm{p}=0,019)$, soziale Internalität $(\mathrm{P})$ (Zeit $\times$ Training: $\mathrm{F}=4,83, \mathrm{df}=2,39$, $\mathrm{p}=0,013)$ und fatalistische Externalität (C) (Zeit: $F=3,33, d f=2,39, p=0,046)$. Für die Sekundär- und die Tertiärskala ergaben sich die folgenden Effekte: Selbstwirksamkeit (SKI) (Zeit $\times$ Training: $\mathrm{F}=7,00$, $\mathrm{df}=2,39, \mathrm{p}=0,003)$ und Externalität $(P C)$ (Zeit: $\mathrm{F}=4,3 \mathrm{O}, \mathrm{df}=2,39, \mathrm{p}=0,020$; Zeit $\times$ Training: $F=3,53, d f=2,39, p=0,039)$, Internalität vs. Externalität (SKI-PC) (Zeit $\times$ Training: $F=6,48, d f=2,39, p=0,004)$. Kontrasttests ergaben eine signifikante Veränderung gegenüber dem Ausgangswert (vor dem Training) bei der Primärskala Internalität (I) 3 Monate nach dem Training; bei allen andere Primär-, Sekundärund auch der Tertiärskala des FKK fanden sich signifikante Veränderungen im Vergleich zum Ausgangswert direkt nach sowie drei Monate nach dem Training $(\mathrm{p}<0,05)$. Während es bei den Primärskalen Internalität $(\mathrm{P})$ und fatalistische Exter- nalität (C) sowie der Sekundärskala Externalität (PC) nach dem Training zu einer Reduktion der Punktwerte kommt, fand sich ein Anstieg bei den anderen Skalen (Selbstkonzept eigener Fähigkeit, soziale Internalität, Selbstwirksamkeit), inklusive der Tertiärskala Internalität vs. Externalität (SKI-PC). Bei den Kontrollprobanden ohne Seminarteilnahme fanden sich keine signifikanten Veränderungen in den Skalenwerten des FKK.

\section{Diskussion}

Die wesentlichen Ergebnisse der dargestellten Untersuchung eines Programms zum „Patientengerechten Umgang mit Aggression in der Psychiatrie“ sind

1. eine sehr gute bis gute Beurteilung des Seminars und der erfragten Teilaspekte durch die Teilnehmer und

2. signifikante Veränderungen im Fragebogen zu Kompetenz- und Kontrollüberzeugungen (FKK), die als Indikator für eine vermehrte internale Kontrollüberzeugung mit relativer Unabhängigkeit von Zufallseinflüssen, geringer Fremdbestimmung, hoher $\mathrm{Au}$ tonomie und aktiv handlungsorientiertem Verhalten angesehen werden können.

Inwiefern das dargestellte Seminar auch zu einer Reduktion von Gewalt und Aggression sowie sich daraus ergebenden Verletzungen führt, wird derzeit am MaxPlanck-Institut für Psychiatrie in München untersucht: In einem Vorher/Nachvergleich werden die Effekte des beschriebenen Trainings in Kombination mit einem neuen Sicherheitskonzept untersucht. Die Datenerhebung ist noch nicht abgeschlossen, da aufgrund der Frequenz erfasster Ereignisse ein ausreichend langer Zeitraum notwendig ist, um mögliche Effekte nachweisen zu können.

Aggression und Gewalt gegen sich oder andere können bei einzelnen Patienten mit psychiatrischen Erkrankungen vorhanden sein und erfordern einen adäquaten Umgang sowie eine Vorbereitung auf solche Situationen. Theoretisches Wissen um Deeskalationsstrategien reicht hierfür nicht aus [Umfrage R. Kuckelsberg, 1999]. Unsere Ergebnisse unterstützen den Nutzen eines praxisorientierten Seminars zum „Patientengerechten Umgang mit Aggression und Gewalt in der Psychiatrie“. Das Programm kann in der Aus- und Weiterbildung unterschiedlicher Berufsgruppen von in der Psychiatrie tätigen Personen genutzt werden. Eine entsprechende Initiative wurde in Ungarn gestartet: Das beschriebene und inzwischen um einen Theorieteil zu Deeskalation erweiterte Programm wird dort im Rahmen der Enthospitalisierungskampagne Mitarbeitern im staatlichen Gesundheitssystem angeboten.

\section{Fazit}

In Ergänzung zu verbalen Deeskalationsstrategien kann ein praxisorientiertes Training den Umgang mit Aggression und Gewalt in der Psychiatrie verbessern und zu stabilen Veränderungen von Kompetenz- und Kontrollerleben bei den Teilnehmern führen. Zur Erhöhung der Sicherheit von Patienten und Mitarbeitern in der Psychiatrie erscheint eine Implementierung in Aus- und Fortbildungen sowie eine breite Anwendung in psychiatrischen Akutkliniken langfristig sinnvoll. Wenn auch durch bisherige Untersuchungen nicht sicher belegt, sollte durch ein praxisorientiertes Training zum Umgang mit Gewalt und Aggression die Zahl [18] und Folgen [5] aggressiver Zwischenfälle in der Psychiatrie reduziert werden können. 


\section{Korrespondenzadresse}

\section{PD Dr. A. Ströhle}

Klinik für Psychiatrie und Psychotherapie,

Charité - Universitätsmedizin Berlin, Campus

Mitte

Charitéplatz 1, 10117 Berlin

andreas.stroehle@charite.de

Interessenkonflikt. Der korrespondierende Autor gibt an, dass kein Interessenkonflikt besteht.

\section{Literatur}

1. Bandura A (1986) Social foundations of thought and action. Prentice-Hall, Engelwood Cliffs, NJ

2. Grainger C, Whiteford $H$ (1993) Assault on staff in psychiatric hospitals: a safety issue. Aust N Z J Psychiatry 27: 324-328

3. Grube M (2001) Aggressivität bei psychiatrischen Patienten. Einflussmöglichkeiten durch ein Selbstschutztraining. Nervenarzt 72: 867-871

4. Haller RM, Deluty RH (1988) Assaults on staff by psychiatric inpatients - a critical review. Br J Psychiatry $152: 174-179$

5. Infantino JA, Musingo SY (1985) Assaults and injuries among staff with and without training in aggression control techniques. Hosp Community Psychiatry 36: 1312-1314

6. Krampen G (1982) Differentialpsychologie der Kontrollüberzeugungen ("Locus of Control"). Hogrefe, Göttingen

7. Krampen G (1991) Fragebogen zu Kompetenzund Kontrollüberzeugungen (FKK). Hogrefe, Göttingen

8. Levenson H (1972) Distinctions within the concept of internal-external control. Proceedings of the Annual Convention of the APA 7: 261-262

9. Levenson $H$ (1974) Activism and powerful others. H Personal Ass 38: 377-383

10. Needham I, Abderhalden C, Meer R et al. (2004) The effectiveness of two interventions in the management of patient violence in acute mental inpatient settings: report on a pilot study. J Psychiatr Ment Health Nurs 11: 595-601

11. Noble P, Rodger S (1989) Violence by psychiatric in-patients. Br J Psychiatry 155: 384-390

12. Richter D (1999) Patientenübergriffe auf Mitarbeiter psychiatrischer Kliniken. Lambertus, Freiburg

13. Richter D, Berger K (2001) Patientenübergriffe auf Mitarbeiter. Eine prospektive Untersuchung der Häufigkeit, Situationen und Folgen. Nervenarzt 72: 693-699

14. Richter D, Needham I (2007) Effects of aggression management trainings for mental health care and disability care staff - a systematic review. Psychiatr Prax 34: 7-14

15. Steinert T, Vogel WD, Beck M, Kehlmann S (1991) Aggressionen psychiatrischer Patienten in der Klinik. Psychiatr Prax 18: 155-161

16. Swanson JW, Swartz MS, Van Dorn RA et al. (2006) A national study of violent behaviour in persons with schizophrenia. Arch Gen Psychiatry 63: 490499

17. Zeller A, Needham I, Halfens R (2006) Effects of training course in management of aggression and violence on nursing students. Pflege 19: 251-258

18. Van Rixtel AM, Nijman HL, Jansen A (1997) Aggression and psychiatry - does training have any effect? Verpleegkunde 12: 111-119

19. Walker Z, Seifert R (1994) Violent incidents in a psychiatric care unit. Br J Psychiatry 194: 826-828

\section{Beeinflusst das Geburtsgewicht den späteren Gemütszustand eines Menschen?}

\section{Eine in der Zeitschrift Biological Psych- iatry veröffentlichte Studie ging der Frage nach, ob ein geringes Geburts- gewicht - ausgelöst durch pränatalen Stress in der Gebärmutter - einen Risi- kofaktor für Depression und Angster- krankungen im späteren Leben dar- stellt.}

Eine Gesundheitsstudie der Universität Cambridge und der Medical School des University College London betrachtete den $\mathrm{Zu}$ sammenhang zwischen dem Geburtsgewicht und der Entstehung von Angsterkrankungen. Dafür wurden Daten der seit 40 Jahre laufenden Erhebung „Medical Research Council National Survey of Health and Development" analysiert, welche 4600 Briten jeweils im Alter von 13, 15, 36, 43 und 53 Jahren auf Symptome von Angst und Depression untersuchte.

Menschen, die in ihrem Leben schon mit psychischen Problemen zu kämpfen hatten, auch wenn es nur leichte bis mittelschwere Symptome von Depression und Angststörungen waren, wiesen ein deutlich geringeres Geburtsgewicht auf. Außerdem erreichten diese Menschen Entwicklungsstadien wie das Stehen und Laufen wesentlich später als mental gesunde Menschen.

Diese Ergebnisse lassen vermuten, dass die Bedingungen im Uterus erheblich auf die weitere Entwicklung eines Kindes einwirken. Steht die Schwangere zum Beispiel unter starkem Stress, führt das zu einer Beeinträchtigung des Blutflusses zur Gebärmutter und somit zur Störung der fetalen Nährstoffzufuhr. Zusätzlich ausgeschüttete Stresshormone gelangen über die Plazenta zum Fetus und lösen dort wahrscheinlich eine falsche Programmierung der für Stressreaktionen verantwortlichen Gehirnbereiche aus. Demnach könnte ein geringes Geburtsgewicht ein Zeichen für pränatalen Stress in der Gebärmutter und eine erhöhte Wahrscheinlichkeit für die Entstehung von Depression und Angststörungen sein.
Originalpublikation:

Colman I, Ploubidis GB, Wadsworth ME, Jones PB, Croudace TJ (2007) A longitudinal typology of symptoms of depression and anxiety over the life course. Biological Psychiatry 62 (Dez.): 1265-1271

Quelle: University of Cambridge, www.cam.ac.uk; University College London Medical School, www.ucl.ac.uk/ medicalschool/index.shtml 